\title{
The van der Waals epitaxial growth of GaSe on Si(111)
}

Le Thanh Vinh

Institute d'Electronique Fondamentale, Université Paris-Sud, URA CNRS n 22, Bâtiment 220,

F-91405 Orsay Cedex, France

M. Eddrief

Laboratoire de Physique des Solides, Associé au CNRS No. 154, Université Pierre et Marie Curie, Tour 13-E2, 4 Place Jussieu, F-75252 Paris Cedex 5, France

John E. Mahan ${ }^{\text {a) }}$ and André Vantomme ${ }^{\text {b) }}$

Instituut voor Kern-en Stralingsfysika, Katholieke Universiteit Leuven, Celestijnenlaan 200 D, B-3001 Leuven, Belgium

J. H. Song ${ }^{\text {c) }}$ and Marc-A. Nicolet

California Institute of Technology, Pasadena, California 91125

(Received 16 July 1996; accepted for publication 24 February 1997)

$\mathrm{GaSe}$, a layered semiconductor, may be grown on the $\mathrm{Si}(111)$ surface by molecular beam epitaxy. The crystalline quality is relatively good, in the sense that the $\mathrm{MeV}^{4} \mathrm{He}$ ion minimum channeling yield $(\sim 30 \%)$ is as low as that of state-of-the-art bulk material, and the interface is atomically abrupt. The initial film deposits are epitaxial islands, and subsequent growth is in the Frank-van der Merwe mode. With the islands already relaxed at the nucleation stage and coalescing to essentially uniform coverage with the first monolayer of deposition, GaSe on $\mathrm{Si}(111)$ provides an example of van der Waals epitaxy. However, it is difficult to understand how epitaxy (crystallographic alignment with the substrate) can occur in such a case, where the film is incommensurate starting from the initial nuclei. A mechanism for alignment of the islands is proposed: they are aligned with the silicon substrate through the influence of dangling bonds at their perimeter, being "quasicommensurate " by virtue of their small lateral size. Although discommensurate regions are created as the islands grow laterally, there is simply no change in their orientation. (c) 1997 American Institute of Physics. [S0021-8979(97)05011-1]

\section{INTRODUCTION}

The hexagonal, layered semiconductor, GaSe, has been shown to grow on $\mathrm{Si}(111)$ by molecular beam epitaxy (MBE) in a remarkable way: the film is crystallographically aligned with the substrate (and remains so after the accumulation of $1 \mu \mathrm{m}$ ) and this epitaxy occurs in spite of the fact that, starting from sub-monolayer coverage, the film is misfitting and relaxed. ${ }^{1}$

This epitaxial growth of $\mathrm{GaSe}$ on $\mathrm{Si}(111)$ is an extension of Koma's original concept of van der Waals epitaxy, the growth of a film on a substrate with very little interaction, in one sense, between the two. ${ }^{2}$ The original implementation of Koma's concept utilized a film and substrate, of which neither presents dangling bonds at their interface. (We infer the following definition of van der Waals epitaxy: epitaxial growth of a film on a substrate when the interfacial forces are of van der Waals-type only.)

The original form of van der Waals epitaxy has been realized with layered semiconductors, such as GaSe, on similarly layered or passivated substrates. With such layered materials, "The atoms in a layer are bound by strong cova-

\footnotetext{
a) Present address: Department of Electrical Engineering, Colorado State University, Fort Collins, CO 80523. Electronic mail: mahan@lance.colostate.edu

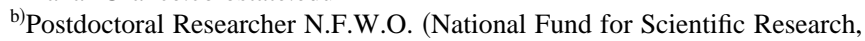
Belgium).

${ }^{c}$ Present address: Advanced Analysis Center, Korea Institute of Science and Technology, PO Box 131, Cheongyang, Seoul 130-650, Korea.
}

lent bonds, whereas the layers are weakly bound to each other by van der Waals forces" 2 and, in van der Waals epitaxy, the films are bound to the substrate in the same weak manner as the layers of the films are bound to each other. The films have been reported to be single crystals even for mismatches well over 10\%. They are deposited by either molecular beam epitaxy (co-evaporation of the elements), or congruent evaporation of the compounds. The films completely wet the substrate (except for pinholes sometimes observed), and a smooth surface is maintained as the thickness is increased. The morphology resembles that of the Frankvan der Merwe growth mode, also known as "layer-bylayer," or "two-dimensional," growth. ${ }^{3}$

Examples include (a) $\mathrm{MoSe}_{2}$ on $\mathrm{SnS}_{2},{ }^{4}$ (b) antimony on mica, ${ }^{5}$ (c) GaSe on $\mathrm{WSe}_{2},{ }^{6}$ (d) $\mathrm{SnS}_{2}$ and $\mathrm{SnSe}_{2}$ on $\mathrm{SnS}_{2}$, $\mathrm{SnSe}_{2}, \mathrm{MoS}_{2}, \mathrm{WSe}_{2}, \mathrm{MoTe}_{2}, \mathrm{GaSe}, \mathrm{InSe}$, and mica, ${ }^{7}$ (e) InSe on $\mathrm{MoTe}_{2},{ }^{8}$ (f) $\mathrm{GaSe}, \mathrm{MoSe}_{2}$, and metal pthalocyanine on sulfur-passivated $\mathrm{GaP}^{9}{ }^{9}$ (g) $\mathrm{MoSe}_{2}$ and $\mathrm{NbSe}_{2}$ on sulfurterminated $\operatorname{GaAs}(111),{ }^{10}$ and $(\mathrm{h})$ metal pthalocyanine on hydrogen-terminated $\mathrm{Si}(111)^{11}$ and (i) on seleniumterminated $\mathrm{GaAs}(111){ }^{12}$

A unique aspect of van der Waals epitaxy is that the films are not subject to coherency strain, and are therefore grown in an elastically relaxed state, even for the first submonolayer of growth. (Indeed, relaxation is to be expected for a relatively hard film which feels a relatively weak binding force to the substrate. ${ }^{13}$ ) This relaxed state of the film nuclei in van der Waals epitaxy is an extremely important point. We will summarize here what is known experimen- 
tally; all observations of this relaxation to date have been made with reflection high energy electron diffraction (RHEED) or low energy electron diffraction (LEED). Le Thanh et al., discussing the GaSe films of this present research in an earlier publication, reported that for even less than one monolayer of deposition (and for thicker deposits as well) "Within the RHEED accuracy (less than 1\%), the film lattice constants parallel to the surface calculated from the streak spacing agree well with those of a GaSe bulk single crystal..." " Koma et al. reported that for selenium grown on tellurium (20\% mismatch), "selenium has its own lattice constant even at the first atomic layer..." ${ }^{2}$ Lang et al. report that a $7 \AA$ GaSe layer grown on $\mathrm{WSe}_{2}$ (14\% mismatch) was fully relaxed. ${ }^{6}$ Schlaf et al. report that a half monolayer of $\mathrm{SnS}_{2}$ grown on cleaved mica (42.9\% mismatch) was relaxed. $^{7}$ Finally, Ohuchi et al. showed that for $\mathrm{MoSe}_{2}$ grown on $\mathrm{SnS}_{2}$ (10\% mismatch), the film lattice constant calculated from the RHEED streak intervals was at least within $1.5 \%$ of the bulk value for $\mathrm{MoSe}_{2}$, for the "deposition of a fraction of one unit layer.', 4

But why is this fully relaxed (yet epitaxial) growth of GaSe on $\mathrm{Si}(111)$, as well as the other examples cited above, so remarkable? Because of the generally anticipated need in epitaxy for a lattice matching between the substrate and the initial film deposits. Batstone states that "...(the incommensurate state) does not seem consistent with the ability to form a unique epitaxially oriented film." 14 One would expect that in foregoing the commensurability of even the initial deposits, we would lose epitaxy in the process. How, then, is alignment achieved in van der Waals epitaxy? But first, how good is the crystallographic alignment for the present case of GaSe on $\operatorname{Si}(111)$ ?

\section{EXPERIMENTAL PROCEDURES}

Our epitaxial GaSe films on $\mathrm{Si}(111)$ were obtained by co-evaporation of gallium and selenium in a conventional MBE system with a base pressure lower than $10^{-10}$ Torr. The two elements were evaporated from Knudsen cells, and their fluxes were monitored by a bare ion gauge placed on the sample manipulator. Following previous optimization studies of GaSe growth, the GaSe films used in this work were grown at substrate temperatures of $500-520^{\circ} \mathrm{C}$ with $\mathrm{Se} / \mathrm{Ga}$ flux ratios of $8-11$. $^{\text {The typical growth rate was }}$ about $10 \AA / \mathrm{min}$, determined by previously counting the number of GaSe layers in cross-sectional transmission electron microscope (TEM) images.

The substrates were $n$-type $(\rho \sim 100 \Omega \mathrm{cm})$ 2-in.-diam $\mathrm{Si}(111)$ wafers. Cleaning of the substrate surface followed the newly developed procedure utilizing the hydrogenterminated $\mathrm{Si}(111)$ surface. ${ }^{15-17}$ It consists of two steps: the first is a wet chemical treatment to prepare an ideally monohydride-terminated $\mathrm{Si}(111)$ surface. The second step is heating in ultrahigh vacuum (at $550{ }^{\circ} \mathrm{C}$ ) to desorb the passivating hydrogen layer.

The RHEED technique was used to monitor the quality of the silicon substrate surface before growth and the structure of the GaSe films during and after growth. Upon desorption of the passivating hydrogen layer from the silicon sub-

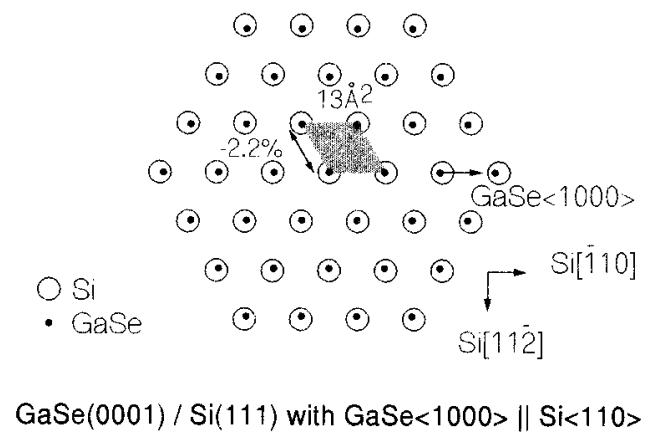

FIG. 1. A proposed lattice matching for GaSe on $\mathrm{Si}(111)$. The room temperature mismatch is $-2.2 \%$, with a common unit mesh area of $13 \AA$ (see Ref. 2). The film is shown relaxed. The epitaxial relationship has been confirmed experimentally.

strates, sharp seventh order streaks were observed in the RHEED patterns.

Phase identification and determination of crystallographic orientation were accomplished with copper $K_{\alpha}$ X-ray diffraction in the $\theta-2 \theta$ geometry. For TEM examination, the cross-sectional samples were prepared first by mechanical thinning and then argon ion milling; images were obtained using $200 \mathrm{kV}$ Jeol $2000 \mathrm{EX}$ and Topcon EM002B electron microscopes.

${ }^{4} \mathrm{He}^{+}$backscattering measurements (for compositional characterization and thickness estimation) and channeling measurements (for characterizing the crystallographic alignment) were made using a scattering angle of $170^{\circ}$. When an optimal mass resolution was desired (hence separating the gallium and selenium signals for thin films), an incoming beam energy of $3.1 \mathrm{MeV}$ was used. On the other hand, when obtaining two-dimensional channeling data for the epitaxial films (for which 400 backscattering spectra were taken-see below), an energy of $600 \mathrm{keV}$ was used. While the mass resolution deteriorates when using this low energy (which causes no problem for channeling since only the total backscattering yield from the gallium and selenium atoms is needed), the scattering cross section increases by a factor of 27. This increase in backscattering yield allows a much shorter measuring time, and hence allows for less irradiation damage to the sample during the critical channeling measurement.

For comparison to the thin film results, measurements were also made on a bulk GaSe single crystal. This sample was grown by the Bridgman technique, which gives samples of the $\epsilon$-GaSe polytype. ${ }^{18}$ It is in the form of a platelet, on the order of $1 \mathrm{~cm}$ in diameter and $1 \mathrm{~mm}$ thick.

\section{THE CRYSTALLOGRAPHIC ALIGNMENT OF GaSe ON Si(111)}

A possible lattice matching for $\mathrm{GaSe}$ on $\mathrm{Si}(111)$ is illustrated in Fig. 1. The epitaxial relationship is

$\operatorname{GaSe}(0001) / \operatorname{Si}(111)$ with $\operatorname{GaSe}\langle 1000\rangle \mid \| \operatorname{Si}\langle 110\rangle$.

There is a hexagonal symmetry match between the matching faces, a $-2.2 \%$ room temperature mismatch (using a GaSe " $a$ " lattice parameter of $3.755 \AA^{19}$ and a silicon surface net parameter, $a / \sqrt{2}$, of $3.8403 \AA^{20}$ ), and a common unit mesh 


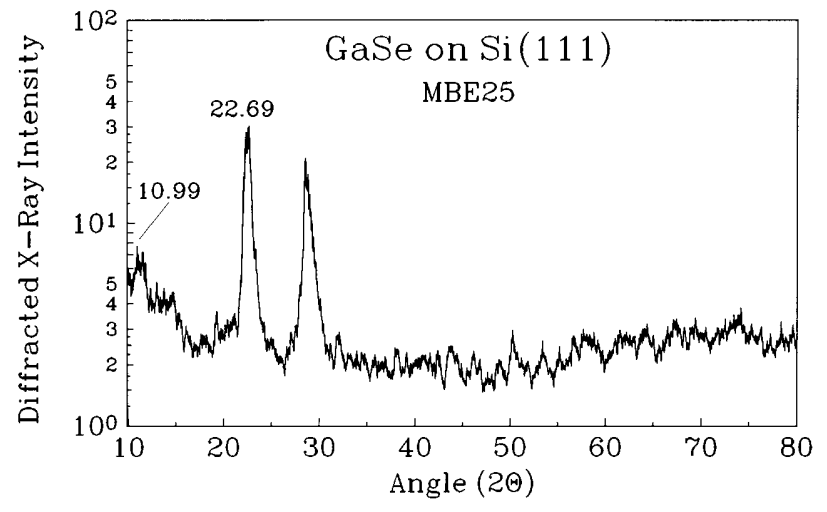

FIG. 2. An x-ray diffraction pattern in the $\theta-2 \theta$ geometry for an epitaxial GaSe film of $115 \AA$ thickness. Copper $K_{a}$ radiation was used. The phase indicated is $\epsilon-\mathrm{GaSe}$ and the matching face is the (0001). The identified peaks are the 0002 and 0004 . The other major peak is due to the silicon substrate.

area of $13 \AA^{2}$. The coincidence net is that of the unreconstructed $\mathrm{Si}(111)$ face. This is, quantitatively, an excellent lattice matching, ${ }^{21}$ and the stated epitaxial relationship was, in fact, observed in the films that we grew.

In Fig. 2 we show an x-ray diffraction pattern for a representative epitaxial film of $115 \AA$ thickness. The two labeled peaks are the $\epsilon$-GaSe 0002 and 0004 . The other significant peak in the pattern is the Si 111, due, of course, to the substrate. The GaSe $c$-lattice parameter calculated from these peak positions is not experimentally distinguishable from that value previously reported for $\epsilon$-GaSe, $15.887 \AA \AA^{22}$ (There are other polytypes of GaSe having different $c$ values. ${ }^{19}$ ) Because the technique measures the interplanar spacing in the direction perpendicular to the substrate surface, the (0001) matching face is established. This result agrees with the epitaxial relationship given above.

Figure 3 shows a two-dimensional channeling pattern for an 840 - $\AA$-thick epitaxial film. The hexagonal symmetry of this pattern clearly demonstrates the GaSe 0001 matching

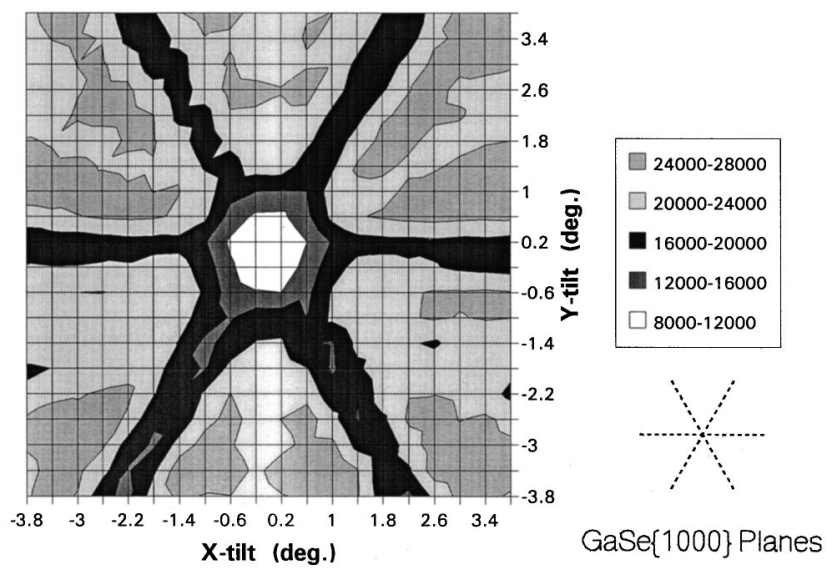

FIG. 3. Two-dimensional channeling pattern for an epitaxial GaSe film 840 $\AA$ thick, taken with a $600 \mathrm{keV}{ }^{4} \mathrm{He}^{+}$analyzing beam. The backscattering yield (number of counts measured) is plotted as a function of the tilt angles in two orthogonal directions (both perpendicular to the sample surface normal). The number of counts is indicated by the gray scale.

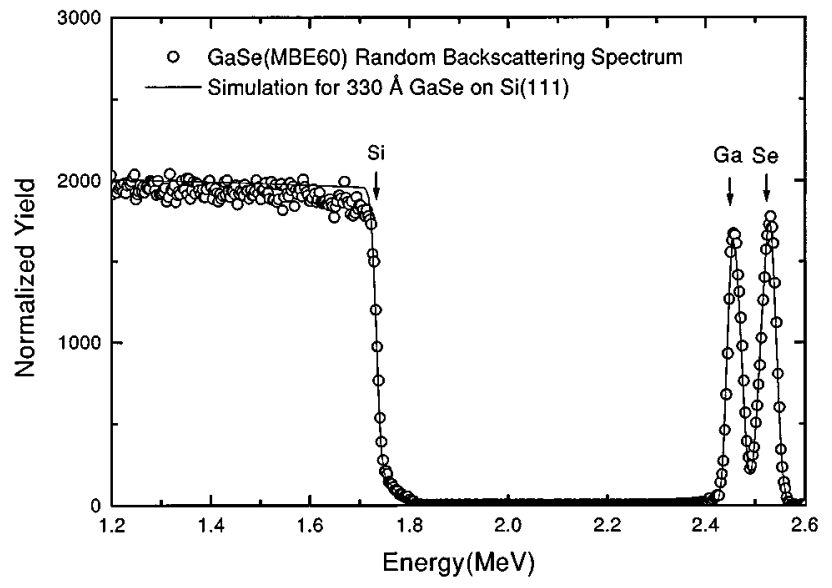

FIG. 4. A backscattering spectrum taken with a $3.1 \mathrm{MeV}^{4} \mathrm{He}^{+}$analyzing beam of random incidence, and RUMP (see Ref. 23) simulation (for the purpose of compositional analysis and thickness estimate) for epitaxial GaSe on $\mathrm{Si}(111)$. The film thickness is $330 \AA$, with no detectable deviation from stoichiometry.

face and the fact that there is only one azimuthal orientation. The deep minima in channeling yield are localized on GaSe $\{1000\}$ planes. A comparison with the two-dimensional channeling pattern for the substrate gave confirmation of the previously stated azimuthal orientation. (The corresponding deep minima for the substrate are localized on $\operatorname{Si}\{110\}$ planes.)

We present in Fig. 4 a backscattering spectrum with a 3.1 $\mathrm{MeV}^{4} \mathrm{He}^{+}$beam incident in a random direction, for another epitaxial film. Within the sensitivity of backscattering spectrometry, no deviation from stoichiometry can be detected. Modeling with RUMP $^{23}$ gave a thickness estimate of $330 \AA$ A. Furthermore, a channeling effect was observed along the direction normal to the substrate surface for films grown under the optimal conditions described above. Figure 5 presents $600 \mathrm{keV}$ backscattering spectra with random and aligned beam incidence for the 840 - $\AA$-thick layer, taken from

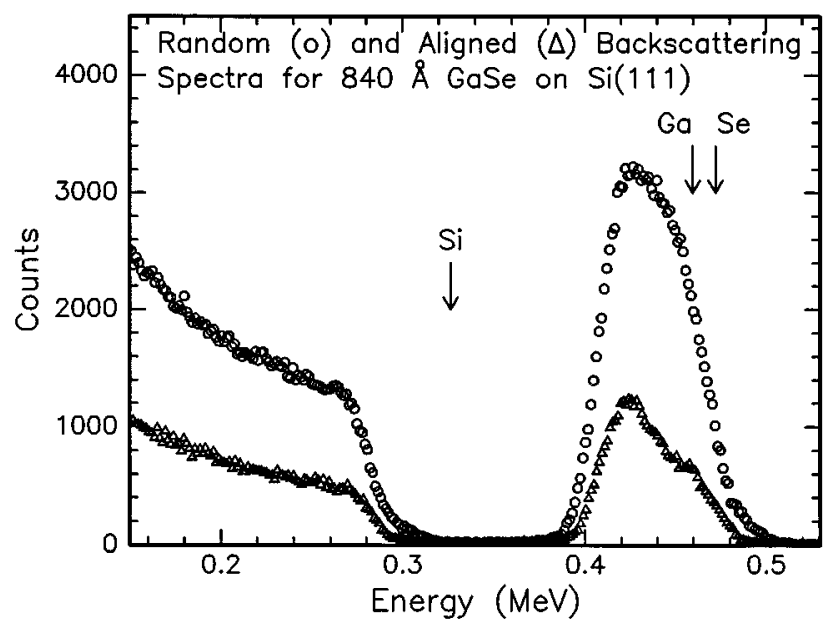

FIG. 5. Backscattering spectra for the same film as in Fig. 3. A $600 \mathrm{keV}$ ${ }^{4} \mathrm{He}^{+}$analyzing beam was used, of random incidence and also aligned along $\mathrm{GaSe}[0001]$. The channeling minimum yield is $\sim 30 \%$. 


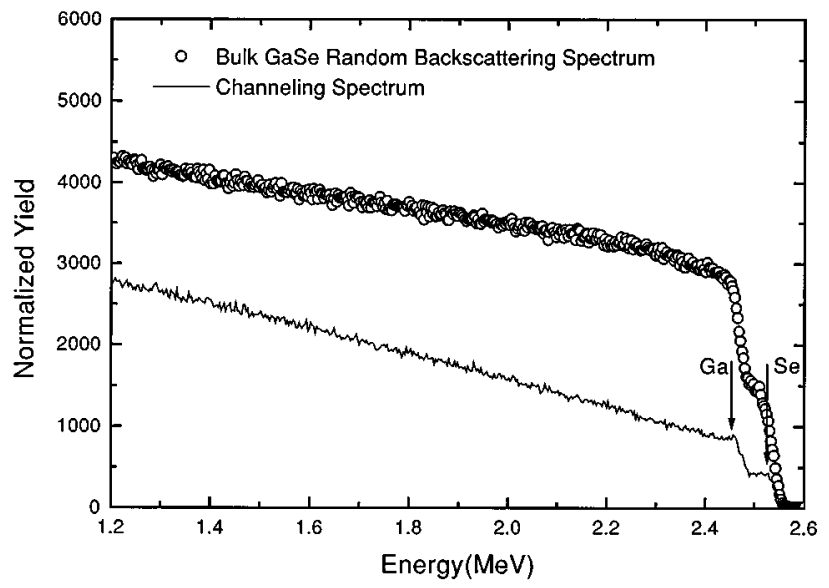

FIG. 6. Backscattering spectra taken with $3.1 \mathrm{MeV}^{4} \mathrm{He}^{+}$analyzing beams of random and aligned incidence, for bulk GaSe with incident beam along [0001]. The minimum channeling yield is $31 \%$.

the two-dimensional image scan in Fig. 3. The channeling minimum yield is $\sim 30 \%$.

The crystallographic orientation of the bulk GaSe platelet (in the direction normal to its flat face) was found to be the same as that of the epitaxial films. (The bulk sample exhibited ten $000 n \mathrm{x}$-ray diffraction peaks attributable to $\epsilon$-GaSe, with $2 \leqslant n \leqslant 11$, which dominate its pattern.) Channeling results for the bulk GaSe crystal are presented in Fig. 6 . The selected channeling axis is perpendicular to the flat face of the crystal, which is parallel to the (0001) planes. The bulk sample presents a minimum channeling yield of $31 \%$.

In Fig. 7 we present a transmission electron microscopy lattice image of a thin film sample, with the incident beam along $\mathrm{Si}\langle 112\rangle$. The $\mathrm{GaSe} / \mathrm{Si}$ interface is abrupt, with no products of a $\mathrm{GaSe}-\mathrm{Si}$ reaction. The observed interplanar spacing of the film $(7.94 \AA$, which is equal to the theoretical $c / 2$ value, to within experimental error) confirms the previously stated matching face. The absence from the micrograph of any tetragonal distortion of the film, or any strain fields in the substrate, suggest a relaxed state for the film. The arrows indicate steps in the substrate surface which caused antiphase boundaries in the film.

We conclude from this broad structural characterization of the epitaxial films that they are single crystalline over a large area, and of a quality at least equal to the best bulk material available. (When nonoptimum growth conditions are employed, there is a deterioration in structural quality of the GaSe film. An example demonstrating the deleterious

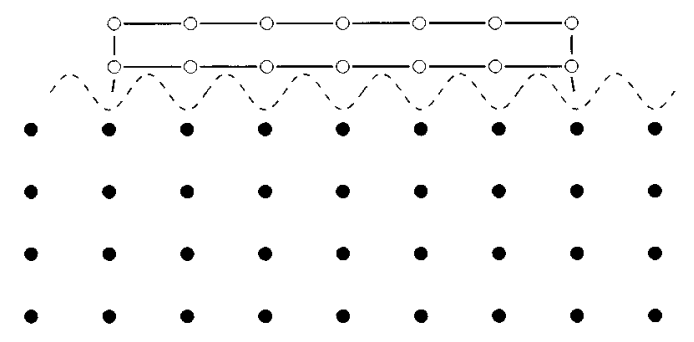

FIG. 8. The concept of a quasi-commensurate, misfitting, relaxed island is illustrated schematically. The solid circles represent atoms of the substrate and the sine wave, the periodic potential energy of adatoms. The open circles represent film atoms in a layered island six atomic spacings wide. The island is shown relaxed, with an assumed mismatch of $-2.2 \%$. The limited lateral size of the island allows it to be quasi-commensurate (without the introduction of misfit dislocations).

effect of an interfacial Si-Se layer is shown in the Appendix.)

\section{A MEANS OF ISLAND ALIGNMENT IN VAN DER WAALS EPITAXY OF GaSe ON Si(111)}

It seems likely that the initial GaSe nuclei on $\mathrm{Si}(111)$ become epitaxially aligned at the time they are formed. But how is this accomplished, if there is only a weak van der Waals interaction at the interface? The $\operatorname{Si}(111)-7 \times 7$ surface would seem as capable as any substrate surface of providing an in-plane directional reference and alignment forces, with its strong, unsaturated bonds. In the dimeradatom-stacking fault (DAS) model, a $\mathrm{Si}(111) 7 \times 7$ surface has 19 dangling bonds per unit cell, and consequently this surface is known to be highly reactive. ${ }^{24}$ Most elemental materials deposited on this surface react with it, resulting in drastic structural and chemical modifications.

While a continuous GaSe layer will have no chemical bonds as such to the substrate, an island will have dangling bonds, at its perimeter. The existence of such dangling bonds was proposed by Lang et al. ${ }^{6}$ They addressed the question of how layer-by-layer growth can occur in a system having such weak interaction between film and substrate. They postulated an anisotropic surface tension for $\mathrm{GaSe}$ - while the surface Gibbs free energy of the van der Waals planes is only $\sim 0.01 \mathrm{eV} /$ atom, that of the edge planes is $\sim 2 \mathrm{eV} /$ atom. Thus, layer-by-layer growth is favored even though the filmsubstrate surface tension is exceedingly low. We now propose that these dangling bonds at the perimeter of the GaSe nuclei interact with those of the silicon substrate to position and align the islands in the observed epitaxial relationship.

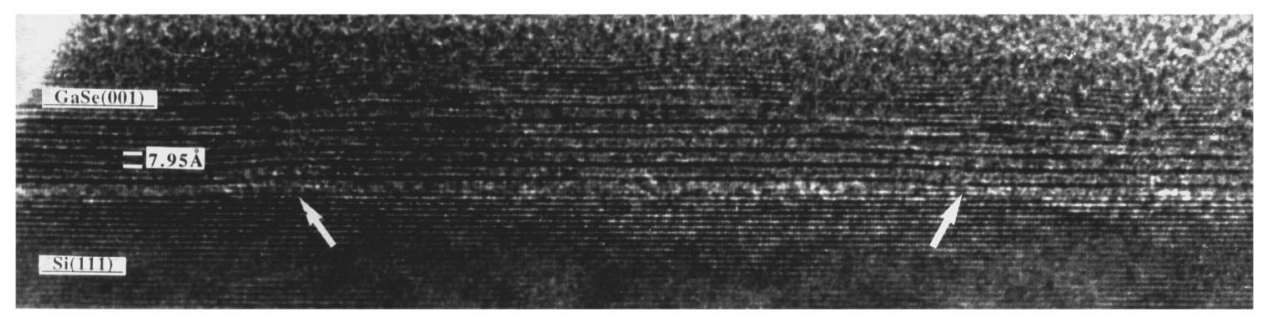

FIG. 7. Transmission electron microscopy lattice image of epitaxial GaSe on $\mathrm{Si}(111)$, with incident beam along $\mathrm{Si}\langle 112\rangle$. 


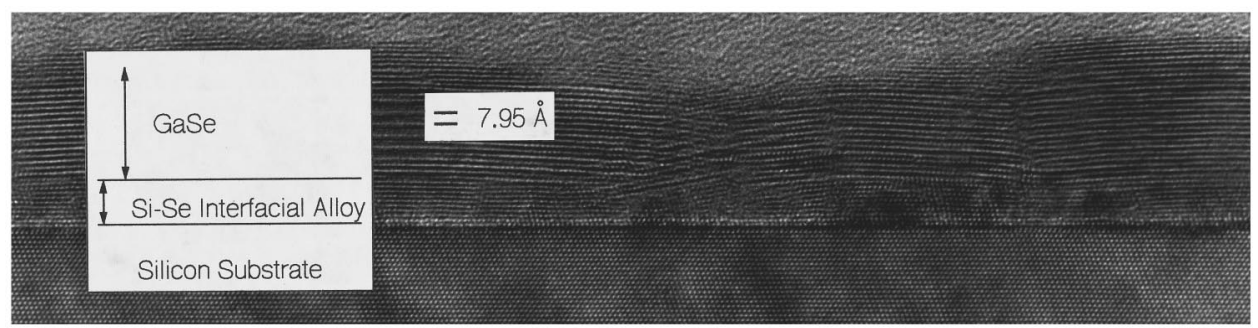

FIG. 9. Transmission electron microscopy lattice image, showing nonepitaxial GaSe grown on a deliberately formed $\mathrm{Si}-\mathrm{Se}$ interfacial layer, on $\mathrm{Si}(111)$. The interfacial layer degrades the epitaxy of GaSe. The incident beam is along $\operatorname{Si}\langle 110\rangle$.

With a modest mismatch at the growth temperature, a relaxed island of limited lateral extent can be "quasicommensurate," i.e., nearly commensurate over limited distances. This idea is illustrated schematically in Fig. 8. As an island grows, its outer regions eventually become incommensurate with the substrate, but there is no reason for the nucleus to change its crystallographic alignment. The first monolayer is formed by the ultimate coalescence of such aligned, relaxed nuclei, resulting in an aligned, relaxed, continuous layer.

Of course, this proposal does not address the larger question of how azimuthal alignment is accomplished in the pure form of van der Waals epitaxy-growth of a layered film upon a layered substrate rather than upon a reactive substrate such as $\mathrm{Si}(111)-7 \times 7$. If an island presents dangling bonds to a passive substrate surface, to what might they attach? Perhaps they are capable of inducing a bond with the substrate at a preferred surface site. To our knowledge, this larger question has never received serious attention.

\section{CONCLUSIONS}

We demonstrate epitaxial growth of the van der Waals crystal, GaSe, on $\mathrm{Si}(111)$. The channeling measurements suggest that a thin GaSe film on silicon is at least equal in crystalline quality to the best available bulk form of GaSe.

This epitaxial system raises several fundamental questions about the mechanisms of alignment. GaSe on Si(111) is an odd case where the interface unit cell is very small and the mismatch is acceptable, but the interface actually possesses no translational periodicity - and yet there is epitaxy!

We hypothesize that dangling bonds at the nucleus perimeters provide the lateral forces for alignment. As the first continuous monolayer is formed, the island perimeters, with their active dangling bonds, disappear. Thus, it is only in the initial island stage of growth when the substrate exerts its influence on the crystallographic alignment of the film.

The most fundamental question is raised by van der Waals epitaxy in its original form, the growth of a layered film upon a passive substrate surface. The several epitaxial systems in this category show that azimuthal alignment does not even depend upon having a chemically active substrate surface. Clearly, van der Waals epitaxy presents challenges to our present understanding of thin film growth.

Finally, GaSe on $\mathrm{Si}(111)$ shows that the van der Waals epitaxy phenomenon does not depend upon having a passive substrate. This goes farther than the observation made by Ohuchi et al., when van der Waals epitaxy on the sulfur- terminated GaAs surface was first demonstrated: “....inertness of the surfaces as well as absence of surface dangling bonds appear to be prerequisites of the [van der Waals epitaxy] process." ${ }^{4}$ We find that van der Waals epitaxy is possible if the film has a layered structure and the substrate is well ordered (but not necessarily also of a layered structure).

\section{ACKNOWLEDGMENTS}

The authors thank James Becker for characterizing the samples by x-ray diffraction, Y. Zheng for TEM characterizations, and A. Chevy for providing bulk GaSe samples. Support from the J. William Fulbright Foreign Scholarship Board, the Research Council of the Catholic University of Leuven, and the Belgian National Science Foundation is gratefully acknowledged.

\section{APPENDIX: THE INFLUENCE OF A SILICON-SELENIUM INTERFACIAL LAYER ON THE EPITAXY OF GaSe}

We show in Fig. 9 a transmission electron microscopy lattice image (beam along $\mathrm{Si}\langle 110\rangle$ ) of a sample specially prepared to reveal the influence of a silicon-selenium interfacial layer on the epitaxy of $\mathrm{GaSe}$ on $\mathrm{Si}(111)$. The preparation was as follows: the $\mathrm{Si}(111)-7 \times 7$ surface was exposed to a flux of selenium at $400-450{ }^{\circ} \mathrm{C}$ until the RHEED pattern became diffuse and unstructured, resembling that of amorphous silicon dioxide. Then, a GaSe film was immediately deposited at a substrate temperature of $450{ }^{\circ} \mathrm{C}$.

In the lattice image of this sample one can clearly identify the silicon-selenium interfacial layer. Moreover, its effect on the subsequently grown GaSe layer is to degrade its crystallographic alignment along the $\mathrm{Si}[111]$ direction and to make this misalignment variable, resulting in tilted domains of GaSe. The same silicon-selenium interfacial layer, and degradation of epitaxy, were observed to a lesser degree in GaSe films deposited directly upon $\operatorname{Si}(111)-7 \times 7$ at $400-$ $450{ }^{\circ} \mathrm{C}$. (Recall that our stated optimum growth temperature was $\left.500-520^{\circ} \mathrm{C}\right)$.

\footnotetext{
${ }^{1}$ V. Le Thanh, M. Eddrief, C. Sébenne, A. Sacuto, and M. Balkanski, J. Cryst. Growth 135, 1 (1993).

${ }^{2}$ A. Koma, K. Sunouchi, and T. Miyajima, J. Vac. Sci. Technol. B 3, 724 (1985)

${ }^{3}$ E. Bauer and H. Poppa, Thin Solid Films 12, 167 (1972).

${ }^{4}$ F. S. Ohuchi, B. A. Parkinson, K. Ueno, and A. Koma, J. Appl. Phys. 68, $2168(1990)$

${ }^{5}$ H. J. Osten, J. Klatt, and G. Lippert, Appl. Phys. Lett. 60, 44 (1992).
} 
${ }^{6}$ O. Lang, R. Schlaf, Y. Tomm, C. Pettenkofer, and W. Jaegermann, J. Appl. Phys. 75, 7805 (1994).

${ }^{7}$ R. Schlaf, D. Louder, O. Lang, C. Pettenkofer, W. Jaegermann, K. W. Nebesny, P. A. Lee, B. A. Parkinson, and N. R. Armstrong, J. Vac. Sci. Technol. A 13, 1761 (1995).

${ }^{8}$ R. Schlaf, S. Tiefenbacher, O. Lang, C. Pettenkofer, and W. Jaegermann, Surf. Sci. Lett. 303, L343 (1994).

${ }^{9}$ C. Hammond, A. Back, M. Lawrence, K. Nebesny, P. Lee, R. Schlaf, and N. R. Armstrong, J. Vac. Sci. Technol. A 13, 1768 (1995).

${ }^{10}$ K. Ueno, T. Shimada, K. Saiki, and A. Koma, Appl. Phys. Lett. 56, 327 (1990).

${ }^{11}$ H. Tada, T. Kawaguchi, and A. Koma, Appl. Phys. Lett. 61, 2021 (1992).

${ }^{12}$ H. Yamamoto, H. Tada, T. Kawaguchi, and A. Koma, Appl. Phys. Lett. 64, 2099 (1994).

${ }^{13}$ J. H. van der Merwe, in Single-Crystal Films, edited by M. H. Francombe and H. Sato (Pergamon, Oxford, 1964), p. 139.

${ }^{14}$ J. L. Batstone, Materials Interfaces-Atomic-level Structure and Properties, edited by D. Wolf and S. Yip (Chapman and Hall, London, 1992), p. 316.
${ }^{15}$ G. S. Higashi, Y. J. Chabal, G. W. Trucks, and K. Raghavachari, Appl. Phys. Lett. 56, 656 (1990).

${ }^{16}$ G. S. Higashi, R. S. Becker, Y. J. Chabal, and A. J. Becker, Appl. Phys. Lett. 58, 1656 (1991).

${ }^{17}$ Le Thanh Vinh, M. Eddrief, C. A. Sebenne, P. Dumas, A. Taleb-Ibrahimi, R. Gunther, Y. J. Chabal, and J. Derrien, Appl. Phys. Lett. 64, 3308 (1994).

${ }^{18}$ A. Kuhn, A. Chevy, and R. Chevalier, Phys. Status Solidi A 31, 496 (1975).

${ }^{19}$ Data in Science and Technology, edited by R. Poerschke and O. Madelung (Springer, Berlin, 1992), p. 34.

${ }^{20}$ In Ref. 19, 1991, p. 15.

${ }^{21}$ A. Zur and T. C. McGill, J. Appl. Phys. 55, 378 (1984).

${ }^{22}$ W. Schubert, E. Dörre, and M. Kluge, Metallkd. 46, 216 (1955).

${ }^{23}$ L. R. Doolittle, Nucl. Instrum. Methods Phys. Res. B 9, 334 (1985).

${ }^{24}$ K. Takayanagi, Y. Tanishiro, M. Takahashi, and S. Takahashi, J. Vac. Sci. Technol. A 3, 1502 (1985). 